\title{
Quality of life of family caregivers of cancer patients in Singapore and globally
}

Haikel A $\underline{\text { Lim }}^{1,2}$, HBSSc, MSc, Joyce YS $\underline{\text { Tan }}^{1}$, HBSSc, Joanne $\underline{\text { Chua }}^{3}$, MSc, MPsy, Russell KL Yoong ${ }^{1}$, Siew Eng $\underline{\text { Lim}}^{4}$, MB BCh BAO, ABM, Ee Heok Kua ${ }^{1,3}$, MD, FRCPsy, Rathi Mahendran ${ }^{1,2,3}$, MBBS, MMed

INTRODUCTION Family caregivers of cancer patients often suffer from impaired quality of life (QOL) due to stress arising from the responsibility of caregiving. Most research on such QOL impairments was conducted in Western populations. Thus, this exploratory study sought to (a) examine the QOL levels of family caregivers of cancer patients in an Asian population in Singapore, in relation to caregivers from other countries within and outside of Asia; and (b) investigate the association between sociodemographic factors and QOL impairments in family caregivers in Singapore.

METHODS A total of 258 family caregivers of cancer patients who were receiving outpatient treatment completed the Caregiver Quality of Life Index-Cancer (CQOLC) and a sociodemographic survey. We compared the published CQOLC total scores from Turkey, Iran, Taiwan, South Korea, the United Kingdom, the United States and Canada with the Singapore dataset and examined the demographic relationships.

RESULTS Caregivers in Singapore and Asia had lower CQOLC total scores than their Western counterparts. Caregivers who were male, of Chinese ethnicity, had parental relationships with their care recipient, or cared for advanced-stage cancer patients were found to have impaired QOL.

CONCLUSION The findings of this study highlight possible areas in which support can be provided for family caregivers of cancer patients, and underscore the need to reconcile cultural diversity, values, societal expectations and demographic characteristics in Singapore.

Keywords: cancer patients, family caregivers, quality of life

\section{INTRODUCTION}

The chronicity of cancer has shifted care to the home, with family members often being the main care providers for cancer patients. ${ }^{(1)}$ While cancer caregiving is a meaningful experience, it is also associated with deteriorating quality of life (QOL), ${ }^{(1)}$ greater psychiatric sequelae, (2) and increased risk of mortality for the caregiver. ${ }^{(3)}$ These effects on caregivers also ultimately affect the quality of care received by cancer patients. ${ }^{(4)}$ The deteriorating QOL among family caregivers of cancer patients may be of significance in an Asian country such as Singapore, where cultural norms of filial piety ${ }^{(5)}$ and societal expectations mandate that family members take on the responsibility of being main caregivers. ${ }^{(6)}$ It is therefore essential to identify, understand and support family caregivers and, by extension, their care recipients. Unfortunately, most of the published studies on the associations between caregivers' QOL and demography have been conducted in Western societies and settings. ${ }^{(7)}$ To the best of our knowledge, there have been no studies conducted in Singapore to identify the type of stress experienced by family caregivers of cancer patients and the needs of these caregivers. This lack of information represents a gap in service provision for this group of caregivers.

The present exploratory study sought to address the paucity of research on the QOL of family caregivers of cancer patients in Singapore by: (a) determining the QOL of such caregivers using objective measures; (b) identifying potential sociodemographic correlates of QOL impairments; and (c) comparing the QOL of such caregivers with that of caregivers from other countries in Asia and beyond. We hypothesised that the QOL impairments faced by family caregivers of cancer patients in Singapore would be comparable to those of caregivers in other countries and that there would be demographic differences in caregiving.

\section{METHODS}

Data was pooled from three cross-sectional, ethics-approved studies (i.e. National University of Singapore's Institutional Review Board and the National Healthcare Group Domain Specific Review Board) conducted in Singapore between 2012 and 2014 (Table I). ${ }^{(5,8,9)}$ The response rates in these three studies ranged from $60 \%$ to $87 \%$ and participants had provided informed consent. Included participants were aged $\geq 18$ years, family caregivers of patients with cancer, and able to communicate in English or Mandarin. Domestic helpers who acted as caregivers and participants with known mental health problems or cognitive impairments were excluded from the study analyses. All participants had been recruited at the waiting area of the outpatient clinic of National University Hospital, Singapore, while they were accompanying their care recipients to their appointments.

Participants completed a sociodemographic questionnaire and the Caregiver Quality of Life Index-Cancer (CQOLC). ${ }^{(10)}$ The CQOLC has the best psychometric properties among all

\footnotetext{
'Department of Psychological Medicine, National University of Singapore, ${ }^{2}$ Duke-NUS Medical School, ${ }^{3}$ Department of Psychological Medicine, National University Hospital, ${ }^{4}$ Department of Haematology-Oncology, National University Cancer Institute, Singapore

Correspondence: A/Prof Rathi Mahendran, Senior Consultant Psychiatrist, Department of Psychological Medicine, National University of Singapore, NUHS Tower Block, Level 9 , 1E Kent Ridge Road, Singapore 119228. rathi_mahendran@nuhs.edu.sg
} 
Table I. Sociodemographic characteristics of the caregivers $(n=258)$.

\begin{tabular}{|c|c|}
\hline Characteristic & No. (\%) \\
\hline \multicolumn{2}{|l|}{ Age group (yr) } \\
\hline $18-20$ & $5(2)$ \\
\hline $21-30$ & $44(17)$ \\
\hline $31-40$ & $56(22)$ \\
\hline $41-50$ & $58(22)$ \\
\hline $51-60$ & $51(20)$ \\
\hline $61-65$ & $34(13)$ \\
\hline$>65$ & $10(4)$ \\
\hline \multicolumn{2}{|l|}{ Gender } \\
\hline Male & $107(41)$ \\
\hline Female & $151(59)$ \\
\hline \multicolumn{2}{|l|}{ Ethnicity } \\
\hline Chinese & $173(67)$ \\
\hline Non-Chinese* & $85(33)$ \\
\hline \multicolumn{2}{|c|}{ Relationship to patient ${ }^{+}(n=257)$} \\
\hline Child & $107(42)$ \\
\hline Spouse & $93(36)$ \\
\hline Others ${ }^{*}$ & $57(22)$ \\
\hline \multicolumn{2}{|c|}{ Education level ${ }^{+}(n=238)$} \\
\hline Primary or lower & $36(15)$ \\
\hline Secondary & $74(31)$ \\
\hline Tertiary & $128(54)$ \\
\hline \multicolumn{2}{|c|}{ Patient illness stage $^{+}(n=257)$} \\
\hline Early & $97(38)$ \\
\hline Advanced & $125(49)$ \\
\hline Unsure & $19(7)$ \\
\hline Remission & $16(6)$ \\
\hline
\end{tabular}

*Includes local minority ethnic groups, such as Malay, Indian and Eurasian. + Total does not add up to 258 because of missing data (determined to be missing at random). Percentages are calculated based on existing data. łIncludes sibling, parent, nephew/niece, daughter/son-in-law and grandchild.

disease-specific QOL measures for cancer caregivers, ${ }^{(11)}$ has been validated in Singapore ${ }^{(5)}$ and is used extensively worldwide. It assesses QOL using a 35-item self-report measure. Each of the 35 items is rated on a five-point Likert-type scale, from 0 (not at all) to 4 (very much), and the items cover four domains: burden, disruptiveness, positive adaptation and financial concerns. The total score was obtained by summing up the scores of all the items (maximum score $=140$ ), with a higher score denoting better QOL. ${ }^{(10)}$ Both the English and Mandarin versions ${ }^{(12)}$ of the CQOLC have been validated for use in Singapore and have demonstrated adequate reliability. A Singapore-specific, factor-structure version known as the CQOLC-S25 has also been developed, in which 25 of the 35 items were clustered into five domains: burden, physical/practical concerns, emotional reactivity, self-needs and social support. ${ }^{(5)}$

To determine the differences between the QOL of caregivers in Singapore and that of caregivers from other countries, a scoping review of the literature was conducted to determine suitable sample populations for comparisons. The PubMed database was searched in May 2015 without language or date restrictions using "(((cancer) AND caregiver) AND quality of life)) AND ((((caregiver quality of life index cancer) OR cqolc*)
OR cqol-c) OR cqol\$c)". Of the 127 articles that were returned from that search, only 66 (52\%) were related to the QOL of caregivers of cancer patients and only 35 (28\%) used the CQOLC. Among the studies that used the CQOLC, 8 (23\%) involved the caregivers of inpatients and 13 (37\%) had missing CQOLC total scores. If there was more than one study from a country reporting mean CQOLC scores, the most recent and most similar study was used for comparison. The final studies used for comparison with the Singapore cohort were from Taiwan, ${ }^{(12)}$ South Korea, ${ }^{(13)}$ Iran, ${ }^{(14)}$ Turkey, ${ }^{(15)}$ the United Kingdom, ${ }^{(16)}$ the United States ${ }^{(17)}$ and Canada. ${ }^{(18)}$

Data was analysed using IBM SPSS Statistics version 20.0 (IBM Corp, Armonk, NY, USA), with the alpha level set at 0.05. Onesample Bonferroni-corrected $t$-tests were employed to compare the CQOLC total score of caregivers from Singapore and that of caregivers from other countries, as the original factor structure has not been internationally validated. Independent sample $t$-tests or analyses of variance with Tukey's honest significant differencecorrected post-hoc analyses were employed to examine the associations between the sociodemographic characteristics of the caregivers and their scores in the domains of the validated CQOLC-S25. ${ }^{(5)}$

\section{RESULTS}

A total of 258 family caregivers were included in the present study. Table I shows the demographic variables of the caregivers. Their mean total CQOLC-S25 score was $60.7 \pm 15.2$. When analysed according to the five domains of the CQOLC-S25, the mean scores for the following domains were - burden: $20.4 \pm 6.9$; physical/ practical concerns: $12.4 \pm 4.5$; emotional reactivity: $6.1 \pm 3.7$; self-needs: $10.7 \pm 3.9$; and social support: $11.1 \pm 3.1$. Analyses revealed that male caregivers had significantly lower CQOLC-S25 scores than female caregivers in the domains of physical/practical concerns $(p=0.004)$ and self-needs $(p=0.032)$. Analyses also showed that the CQOLC-S25 scores of Chinese caregivers (as compared to non-Chinese caregivers), caregivers of parents (as compared to those caring for spouses) and caregivers of advancedstage cancer patients (as compared to those caring for early-stage cancer patients) were significantly lower in the social support domain ( $p=0.007, p=0.024$ and $p=0.028$, respectively). The caregivers of advanced-stage cancer patients, however, reported significantly better QOL in the burden domain as compared to the caregivers of early-stage cancer patients $(p=0.038)$. Apart from those mentioned, no other demographic variables were associated with significantly lower QOL scores.

Using the 35-item CQOLC, the mean total CQOLC score for our study cohort was $83.5 \pm 19.1$ (range 22.3-131.4). This score was compared with the scores of cohorts from other countries. Family caregivers of cancer patients in Singapore were found to have significantly impaired QOL as compared to caregivers in the West (i.e. the United Kingdom, the United States and Canada) (Table II). Among the Asian countries, family caregivers of cancer patients in Singapore were found to have similar QOL to caregivers in Turkey and Taiwan, and significantly better QOL than caregivers in Iran and South Korea. 
Table II. Comparison of mean total CQOLC scores of family caregivers of cancer patients in Singapore and other countries.

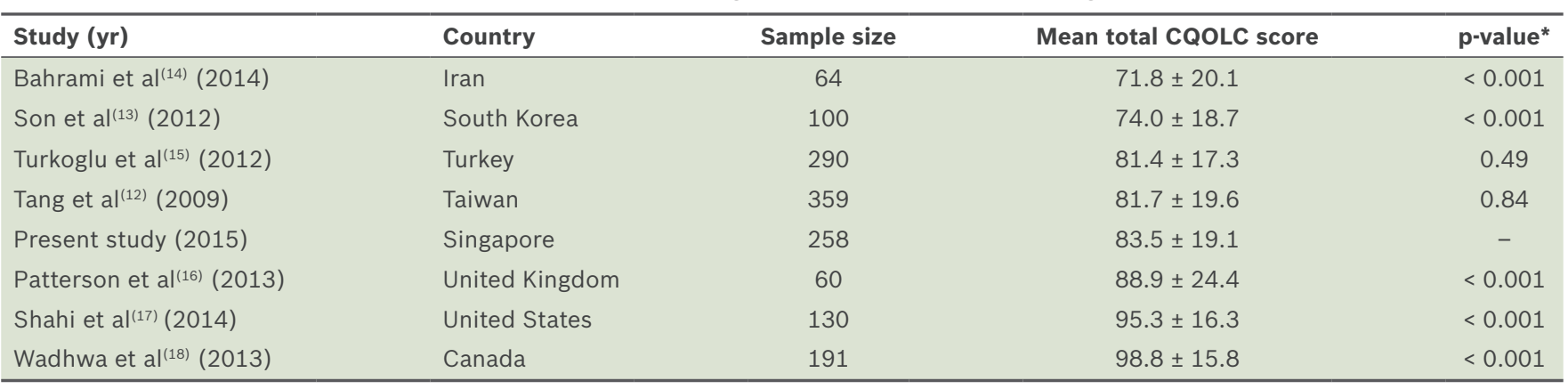

*Bonferroni-corrected $\mathrm{p}$-values based on $t$-tests comparing the mean total CQOLC score of the Singapore cohort to that of other countries. CQOLC: Caregiver Quality of Life Index-Cancer

\section{DISCUSSION}

In the present study, we found that the caregivers of cancer patients in Singapore and other Asian countries have an impaired QOL relative to their counterparts in Europe or America. The caregiving experience within the family depends not only on available resources and caregiving demands, but also on existing family dynamic systems, broader sociocultural and religious beliefs, and the caregiver's resilience and capacity to withstand crises, adapt and cope. ${ }^{(19)}$ The findings of the present study likely reflect the vast differences among respondents from the various countries. While cross-cultural differences were not explored, the available literature on caregiving highlights themes of filial piety and obligatory care as motivations for caregiving, , $^{20,21}$ particularly in Asian (e.g. Singapore, Taiwan and Korea) and Muslim (e.g. Turkey and Iran) societies.

Similarly, caregivers of other clinical populations (e.g. patients with dementia) also appeared to be influenced by notions of filial piety and obligatory care. A study investigating the factor structure of the Zarit Burden Interview among Singaporean caregivers of dementia patients identified a unique dimension described as 'worry about caregiver performance', (22) suggesting the presence of expectations for the standard of care provided among Asian caregivers. In Malaysia, a country that is culturally similar to Singapore, it was found that having formal support in caregiving (such as having a domestic maid or a private nurse) did not alleviate the burden of family caregivers who were caring for dementia patients, emphasising the value of providing familial care. ${ }^{(23)}$

It is also possible that Asian patients experience more severe symptoms of emotional stress and unmet needs ${ }^{(24)}$ as compared to their Western counterparts or the general population. ${ }^{(25)}$ This may influence the caregiver's emotional well-being and result in QOL impairments, as it exacerbates the negative perception of providing care and increases role strain. ${ }^{(26)}$ Furthermore, the Asian societies included in the present study were rapidly developing post-industrial societies, whose populations tend to have poorer work-life balance and increased stressors; these factors may have affected both the patients and their caregivers.

In the present study, we found that the age and educational level of the family caregivers in Singapore were not significantly associated with their QOL. These findings are in contrast to those of other studies conducted globally, which suggested the influence of these predictors. ${ }^{(2,27,28)}$ Given that Singapore's healthcare model is based on enforced healthcare savings, such proxies of socioeconomic status may not necessarily be a significant determinant of QOL; however, it should be noted that the influence of income on caregiver QOL was not captured in the present study. Family caregivers of patients with advancedstage cancer understandably experienced impaired QOL in the domains of burden and social support when compared to those caring for patients with early-stage cancer. Advanced-stage cancers may emphasise the difficult realities of palliative care and the patient's mortality, which can induce stress in family members who are involved in care planning and end-of-life issues. It was also understandable that parents who also acted as caregivers were found to have impaired QOL in the domain of social support, given that their own children were likely unable to assist them with caregiving.

We also found that male family caregivers of cancer patients in Singapore had more impaired QOL than their female peers in the domains of physical/practical concerns and self-needs. This finding is in contrast to those of other studies that reported lower QOL among women due to their traditional gender role. ${ }^{(29)}$ In Singapore, as in many Asian societies, the responsibility of family caregiving traditionally falls on women, while men are expected to provide for the family. Thus, it seems that some men (i.e. sons or husbands) may experience additional role strain when they are forced to balance both the home and work spheres to care for a relative with cancer.

Research on interventions for family caregivers has shown that existential therapy focusing on the development of hope ${ }^{(30)}$ and home care services ${ }^{(31)}$ is effective in supporting caregivers of care recipients who have advanced cancer and palliative care needs. The findings of a previous study on communication skills training for spousal caregivers of breast cancer patients ${ }^{(32)}$ suggest that such training may be a useful means of support for male caregivers. While studies on interventions that cater to spousal caregivers have been published, ${ }^{(32)}$ to our best knowledge, no studies on interventions catering specifically to caregivers caring for their parents have been conducted.

The results of the present study should be interpreted with caution, given that they were obtained from exploratory secondary analyses of primary data. While every effort was made to ensure the similarity of the sample populations in the selected 
studies, various disease and health characteristics that cannot be controlled for are inherent in all mixed-cancer cohort research. In addition, potentially confounding data on the caregiver's health, wealth and employment status was not captured or controlled for. Another possibility for the impaired QOL among Asian caregivers could be the CQOLC itself; cross-cultural validations of the CQOLC in each of the Asian countries have suggested that a different factor structure underlies disease-specific caregiver QOL, with poor model fit and internal reliability for the overarching latent CQOLC. Different aspects of caregiving may therefore contribute to overall disease-specific QOL. However, as inherent in all cross-cultural research, there remain cultural variations that cannot be adequately captured, ${ }^{(33)}$ even with more global or general health-related QOL instruments. ${ }^{(11)}$

To conclude, the findings of the present study provide an avenue for future research into the association of demographic characteristics with caregiver QOL within a particular cultural context. As societal expectations and beliefs differ across cultures, the implications of these varying cultural features (which operate through diverse demographic characteristics) on caregiver QOL deserve greater attention.

\section{REFERENCES}

1. Kim Y, Given BA. Quality of life of family caregivers of cancer survivors: across the trajectory of the illness. Cancer 2008; 112(11 Suppl):2556-68.

2. Given B, Wyatt G, Given C, et al. Burden and depression among caregivers of patients with cancer at the end of life. Oncol Nurs Forum 2004; 31:1105-17.

3. Schulz R, Beach SR. Caregiving as a risk factor for mortality: the Caregiver Health Effects Study. JAMA 1999; 282:2215-9.

4. Chang YJ, Kwon YC, Lee WJ, et al. Burdens, needs and satisfaction of terminal cancer patients and their caregivers. Asian Pac J Cancer Prev 2013; 14:209-16.

5. Mahendran R, Lim HA, Chua J, et al. The Caregiver Quality of Life Index-Cance (CQOLC) in Singapore: a new preliminary factor structure for caregivers of ambulatory patients with cancer. Qual Life Res 2015; 24:399-404.

6. Leow MQ, Chan MF, Chan SW. Predictors of change in quality of life of family caregivers of patients near the end of life with advanced cancer. Cancer Nurs 2014; 37:391-400.

7. Chambers SK, Hyde MK, Au AM, et al. A systematic review of psycho-oncology research in Chinese populations: emerging trends. Eur J Cancer Care (Engl) 2013; 22:824-31.

8. Mahendran R, Ng HY, Chua J, et al. A mixed-methods investigation into the efficacy of a psycho-educational support group therapy for family caregivers of cancer patients: The COPE study overview. Ann Acad Med Singapore 2014; 43:S45-S46.

9. Mahendran R, Lim HA, Tan JY. The relationship between hope, resilience, optimism, and psychiatric co-morbidities in cancer patients: The HOPE study overview. Ann Acad Med Singapore 2014; 43:S45.

10. Weitzner MA, Jacobsen PB, Wagner H Jr, Friedland J, Cox C. The Caregiver Quality of Life Index-Cancer (CQOLC) scale: development and validation of an instrument to measure quality of life of the family caregiver of patients with cancer. Qual Life Res 1999; 8:55-63.

11. Edwards B, Ung L. Quality of life instruments for caregivers of patients with cancer: a review of their psychometric properties. Cancer Nurs 2002; 25:342-9.

12. Tang WR, Tang ST, Kao CY. Psychometric testing of the Caregiver Quality Of Life Index-Cancer on a Taiwanese family caregiver sample. Cancer Nurs 2009; 32:220-9.

13. Son KY, Lee CH, Park SM, et al. The factors associated with the quality of life of the spouse caregivers of patients with cancer: a cross-sectional study. J Palliat Med 2012; 15:216-24.

14. Bahrami M, Farzi S. The effect of a supportive educational program based on COPE model on caring burden and quality of life in family caregivers of women with breast cancer. Iran J Nurs Midwifery Res 2014; 19:119-26.

15. Turkoglu N, Kilic D. Effects of care burdens of caregivers of cancer patients on their quality of life. Asian Pac J Cancer Prev 2012; 13:4141-5.

16. Patterson JM, Rapley T, Carding PN, Wilson JA, McColl E. Head and neck cancer and dysphagia; caring for carers. Psychooncology 2013; 22:1815-20.

17. Shahi V, Lapid MI, Kung S, et al. Do age and quality of life of patients with cancer influence quality of life of the caregiver? J Geriatr Oncol 2014; 5:331-6.

18. Wadhwa D, Burman D, Swami N, et al. Quality of life and mental health in caregivers of outpatients with advanced cancer. Psychooncology 2013; 22:403-10

19. Baider L. Cancer: A Family Affair. In: Wise TN, Biondi M, Constantini A, eds. Psycho-Oncology. Arlington, VA: American Psychiatric Publishing, 2013: 33-56.

20. Gupta R, Pillai VK. Caregiver burden in South Asian families. J Ethn Cult Divers Soc Work 2000; 9:41-53.

21. Wong OM, Chau $\mathrm{BH}$. The evolving role of filial piety in eldercare in Hong Kong. Asian J Soc Sci 2006; 34:600-17.

22. Lim WS, Cheah WK, Ali N, et al. Worry about performance: a unique dimension of caregiver burden. Int Psychogeriatr 2014; 26:677-86.

23. Choo WY, Low WY, Karina R, et al. Social support and burden among caregivers of patients with dementia in Malaysia. Asia Pac J Public Health 2003; 15:23-9.

24. Mahendran R, Lim HA, Chua J, Lim SE, Kua EH. Psychosocial concerns of cancer patients in Singapore. Asia Pac J Clin Oncol 2017; 13: e96-e103.

25. Yang YL, Liu L, Wang Y, et al. The prevalence of depression and anxiety among Chinese adults with cancer: a systematic review and meta-analysis. BMC Cancer 2013, 13:393.

26. Kim Y. Cancer caregivership. In: BI Carr, Steel J, eds. Psychological aspects of cancer. New York, NY: Springer, 2013: 213-20.

27. Hagedoorn M, Sanderman R, Bolks HN, Tuinstra J, Coyne JC. Distress in couples coping with cancer: a meta-analysis and critical review of role and gender effects. Psychol Bull 2008; 134:1-30.

28. Goldstein NE, Concato J, Fried TR, et al. Factors associated with caregiver burden among caregivers of terminally ill patients with cancer. J Palliat Care 2004; 20:38-43.

29. Matthews BA. Role and gender differences in cancer-related distress: a comparison of survivor and caregiver self-reports. Oncol Nurs Forum 2003; 30:493-9.

30. Applebaum AJ, Breitbart W. Care for the cancer caregiver: a systematic review. Palliat Support Care 2013; 11:231-52.

31. Harding R, Higginson IJ. What is the best way to help caregivers in cancer and palliative care? A systematic literature review of interventions and their effectiveness. Palliat Med 2003; 17:63-74.

32. Kauffmann R, Bitz C, Clark K, et al. Addressing psychosocial needs of partners of breast cancer patients: a pilot program using social workers to improve communication and psychosocial support. Support Care Cancer 2016; 24:61-5.

33. Baider L, Goldzweig G. Exploration of family care: a multicultural approach. In Grassi L, Riba M, eds. Clinical psycho-oncology: an international perspective. Hoboken, NJ: Wiley-Blackwell, 2012: 187-98. 